\title{
Enhancement of wanlongmycin production by nitrogen ion beam implantation
}

\author{
Xiaoyong Zhang \\ Plant Protection Research Institute \\ Guangdong Academy of Agricultural Science and \\ Chengdu Institute of Biology \\ Chinese Academy of Sciences \\ PR China \\ Birun Lin* \\ Plant Protection Research Institute \\ Guangdong Academy of Agricultural Science \\ Guangzhou 510640, PR China \\ E-mail: linbr@126.com \\ Xiangyang Gao \\ College of Life Science \\ South China Agricultural University \\ PR China \\ Huifang Shen \\ Plant Protection Research Institute \\ Guangdong Academy of Agricultural Science \\ PR China
}

Financial support: National Natural Science Foundation of China (No. 30471161) and the Guangdong Natural Science Foundation of China (No. 8151064001000010 and No.06025388).

Keywords: mutagen nitrogen ion beam implantation, Streptomyces griseovariabilis GAAS2057, wanlongmycin.

\begin{abstract}
Abbreviations: GAAS: Guangdong Academy of Agricultural Science HPLC: high performance liquid chromatography $\mathrm{KeV}$ : kilo electron volts
\end{abstract}

In an attempt to obtain an industrial strain with higher yield of wanlongmycin, the wild strain Streptomyces griseovariabilis GAAS2507 was mutated by a novel mutagen, nitrogen ion beam with energy of 20 kilo electron volts $(\mathrm{KeV})$ and dose ranging from $7.80 \times 10^{14}$ to $2.86 \times 10^{15}$ ions $/ \mathrm{cm}^{2}$. One mutant strain WN939 was obtained. Its yield of wanlongmycin reached 271.24 $\mu \mathrm{g} / \mathrm{mL}$, which was $82.10 \%$ higher than that of the wild strain. The mutant strain WN939 was relatively stable for the production of wanlongmycin through six successive transfers of cultures and a repeat fermentation in a $30 \mathrm{~L}$ fermentor. In addition, the mutant strains were investigated and divided into five types by their colony phenotypes and production of wanlongmycin. Among them, three types mutant strains exhibited positive mutation, while the other two types mutant strains exhibited negative mutation.

Many agricultural losses are due to plant diseases, insect pests and herb pest; bio-pesticide has become a tendency and a goal of global pesticide development because of its relatively low side-effects and friendliness to environment (Liu and Yao, 2004). In 1996, a new actinomycete strain was isolated by our laboratory from a soil sample collected in Malaysia. It could produce a novel agricultural antibiotic which had a strong activity against a lot of plant oomyces diseases. The novel agricultural antibiotic was named wanlongmycin and believed to be a new member of quinoxaline-like antibiotics by structural identification. Due to low productivity of wanlongmycin, it is urgent that the original strain should be mutated and screened to obtain an industrial high-yield strain.

Muller (1927), the father of radiation genetics, found that $\mathrm{X}$-ray radiation could increase mutation rate. After the ground breaking work of Muller, a great number of studies on mutagenic methods have been established and developed for microbe, plant and animal breeding, including $\gamma$-rays, ultraviolet, alpha particles, beta particles, neutron, etc. However more efforts are still being tried to search for better methods with a higher mutation rate and wider mutation spectra. In 1989, the mutagenicity of low

*Corresponding author 
energy ions on rice was firstly reported in China (Yu et al. 1989). Through exploring the mutagenic effect for years, it was found that this approach possesses a higher mutation rate and a wider mutational spectrum with a higher survival rate (Yu, 1999; Yang et al. 2007). In the study of the mutational mechanisms of low energy ion beam radiation, researchers had proposed that the interaction between low energy ion beam and the organism included energy deposition, momentum transferring, mass deposition and charge exchange (Yu, 2000). Low energy ion beam had a wide range of biological effects, including extracellular and intracellular DNA damage (Zhao et al. 2003), amino acid and nucleotide decomposition (Shi et al. 2001), inactivation of viruses and microbes (Du et al. 1999) and aberrant chromosomes in wheat (Wu and $\mathrm{Yu}, 2001$ ). Although these propositions are still not completely understood, and much research has been studied in depth, the applications of low energy ion beam implantation are to be expanded to serve effectively and profitably for developments of agriculture and biotechnical industries ( $\mathrm{Gu}$ et al. 2008). Since the 1980s, several new varieties and more than 3000 breeding materials have been bred by ion beam implantation in China (Xu et al. 2004). However, ion beam implantation is used in mutational breeding of wanlongmycin-producing micro-organisms for the first time.

Here, we mainly investigated the mutagenic effect of nitrogen ion beam implantation on improving the production of wanlongmycin by Streptomyces griseovariabilis GAAS2507.

\section{MATERIALS AND METHODS}

\section{Strains}

Wanlongmycin-producing strain Streptomyces griseovariabilis GAAS2507 (which is abbreviated to strain

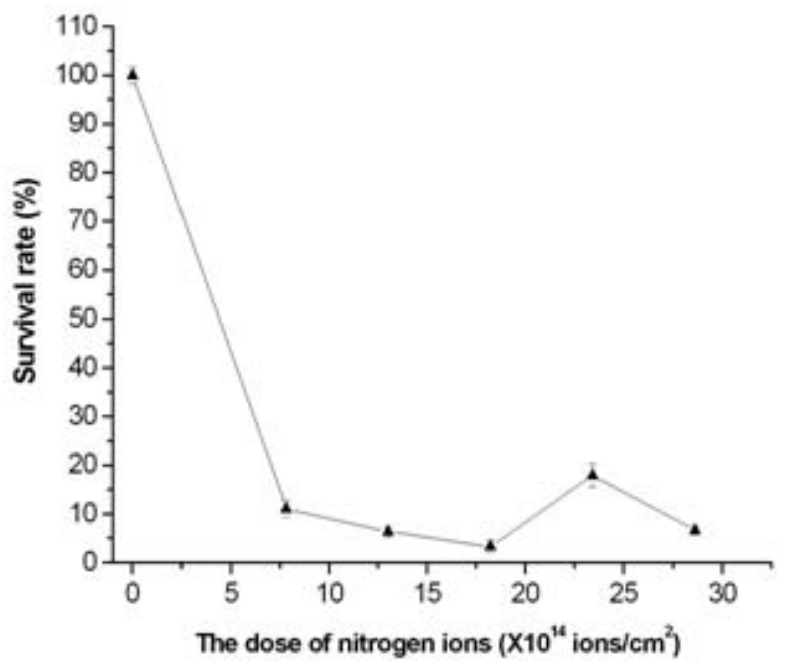

Figure 1. Effect of dose of nitrogen ions on GAAS2507 survival rate. Data were pooled from three independent experiments. Error bars indicate SEs of mean value.

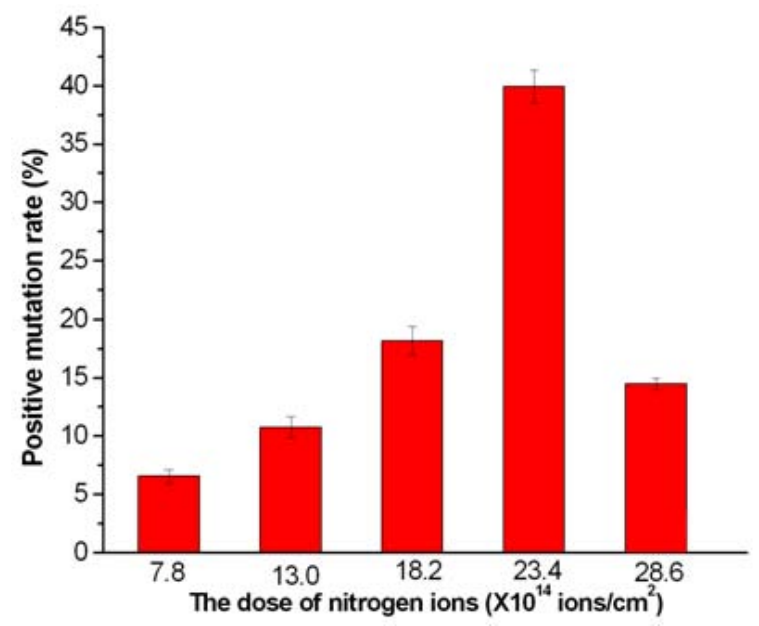

Figure 2. Positive mutation rate of GAAS2507 exposed to nitrogen ions with $20 \mathrm{KeV}$. Data were pooled from three independent experiments. Error bars indicate SEs of mean value.

GAAS2507) is described here and has been deposited in the China Center for Type Culture Collection (CCTCC). Bacillus subtilis MIG1.22 was obtained from the Plant Protection Research Institute, Guangdong Academy of Agricultural Science, China.

\section{Media}

Seed medium: soluble starch $2 \%, \mathrm{KNO}_{3} 0.1 \%, \mathrm{~K}_{2} \mathrm{HPO}_{4}$ $0.05 \%, \mathrm{NaCl} 0.05 \%, \mathrm{FeSO}_{4}$ 0.001\%, $\mathrm{MgSO}_{4} \cdot 7 \mathrm{H}_{2} \mathrm{O} 0.005 \%$, agar $1.5 \%$, pH 7.2.

Fermentation medium: glucose $1 \%$, corn syrup 1.5\%, peptone $0.5 \%, \mathrm{NaCl} 0.5 \%, \mathrm{CaCO}_{3} 0.05 \%,\left(\mathrm{NH}_{4}\right)_{2} \mathrm{SO}_{4}$ $0.05 \%, \mathrm{pH} 7.2$.

BS medium for growth of $B$. subtilis: glucose $0.3 \%$, peptone $0.3 \%, \mathrm{~K}_{2} \mathrm{HPO}_{4} 0.4 \%$, agar $1.5 \%$, $\mathrm{pH} 7.2$.

\section{Culture condition}

Strain GAAS2507 and its mutant strains that had been incubated on seed medium at $28^{\circ} \mathrm{C}$ for 10 days were inoculated from the seed medium to a $500 \mathrm{~mL}$ flask containing $100 \mathrm{~mL}$ of fermentation medium and incubated in a rotary shaker at a speed of $200 \mathrm{r} / \mathrm{min}$ at $32^{\circ} \mathrm{C}$ for 5 days.

The mutant strain with the highest yield of wanlongmycin, after incubation on seed medium at $28^{\circ} \mathrm{C}$ for 10 days, was inoculated from seed medium to a $30 \mathrm{~L}$ jar fermentor (Biostat B) containing $20 \mathrm{~L}$ of fermentation medium, and incubated at $30^{\circ} \mathrm{C}$ for $72 \mathrm{hrs}$, with an aeration rate of 0.6$0.8 \mathrm{vvm}$ and agitation at 300-500 r/min.

B. subtilis was incubated on BS medium at $37^{\circ} \mathrm{C}$ for $12 \mathrm{hrs}$. 
Table 1. The correlation between the colony morphology and production of wanlongmycin of wild and mutant type strains.

\begin{tabular}{|c|c|c|}
\hline $\begin{array}{l}\text { The type of } \\
\text { strain }\end{array}$ & Colony morphology of strain & $\begin{array}{c}\text { Wanlongmycin } \\
\text { concentration } \\
(\mu \mathrm{q} / \mathrm{ml})\end{array}$ \\
\hline Wild type strain & small, transparent, slippery, and did not produce any colored matter or spores & $125.67 \sim 148.95$ \\
\hline $\begin{array}{c}\text { Mutant type A } \\
\text { strain }\end{array}$ & large, transparent, slippery, and did not produce any coloring matter or spores & $162.14 \sim 271.24$ \\
\hline $\begin{array}{c}\text { Mutant type B } \\
\text { strain }\end{array}$ & large, coarse, and produced yellow matter and a few spores & $155.68 \sim 238.98$ \\
\hline $\begin{array}{c}\text { Mutant type C } \\
\text { strain }\end{array}$ & large, coarse, and produced no colored matter but many spores & $146.26 \sim 196.28$ \\
\hline $\begin{array}{l}\text { Mutant type D } \\
\text { strain }\end{array}$ & large, coarse, and produced no colored matter and a few spores & $105.60 \sim 148.12$ \\
\hline $\begin{array}{c}\text { Mutant type E } \\
\text { strain }\end{array}$ & large, coarse, and produced little colored matter and many spores & $67.87 \sim 126.35$ \\
\hline
\end{tabular}

Fifty strains of every type strain were investigated to ascertain the wanlongmycin production of wild type strain and mutant type strains.

\section{Nitrogen ion beam implantation}

Implantation sources were produced by the ion beam bioengineering instrument that was devised by the Chinese Academy of Sciences, Institute of Plasma Physics.

Samples (0.1 mL) of strain GAAS2507 spore suspension (1 x $10^{8}$ spores $/ \mathrm{mL}$ ) were spread to form a single-cell layer on a sterile plate and dried in an extraction hood. The plate was implanted by the nitrogen ion beam with energy of 20 kilo electron volts $(\mathrm{KeV})$ and different doses $\left(7.80 \times 10^{14}\right.$ $2.86 \times 10^{15}$ ions $/ \mathrm{cm}^{2}$ ). The operating pressure in the target chamber was about $10^{-3} \mathrm{~Pa}$. The plates were placed in pairs sequentially in the target chamber. The upper plate was exposed to ion beam implantation, while the lower plate was unexposed and treated as a control. After implantation, every treated plate and control plate were washed with 1 $\mathrm{mL}$ of sterile distilled water (Liu et al. 2006).

\section{Extraction and determination of wanlongmycin}

Extraction and determination of wanlongmycin in fermentation broth had been described by Zhang et al. (2006). Briefly, the cultures were centrifuged at 10000 $\mathrm{r} / \mathrm{min}$ for $10 \mathrm{~min}$ to remove supernatant, and the cells were washed with distilled water three times, then the washed cells were extracted with $60 \%$ aqueous acetone solution for 2 hrs. After extracted solution was centrifuged at 10000 $\mathrm{r} / \mathrm{min}$ for $10 \mathrm{~min}$, the supernatant was filtrated by $0.45-\mu \mathrm{m}$ pore-size millipore filter and was injected into a HPLC
(Symmetry $5 \mu \mathrm{m} \mathrm{C}_{18}$ column, 3.9 x $150 \mathrm{~mm}$, Waters, USA). The mobile phase was methanol-water 20:80 (v/v) which was pumped at a flow rate of $1.0 \mathrm{ml} / \mathrm{min}$ and the elution pattern was monitored at $280 \mathrm{~nm}$.

\section{RESULTS AND DISCUSSION}

\section{Survival rate determination}

Both the dose and the energy level of ion implantation influenced the mutation spectra, and different microorganisms were sensitive to different combinations of these two parameters (Zhou et al. 2005). Strain GAAS2507 was implanted by the nitrogen ion beam with energy of $20 \mathrm{KeV}$ and different doses $\left(7.80 \times 10^{14}, 1.30 \times 10^{15}, 1.82 \times 10^{15}\right.$, $2.34 \times 10^{15}$, and $2.86 \times 10^{15}$ ions $/ \mathrm{cm}^{2}$ ). Figure 1 was the curve of relationship between the survival rate of GAAS2507 and the dose of nitrogen ion beam implantation. When the implantation of dose lower than $1.82 \times 10^{15}$ ions $/ \mathrm{cm}^{2}$, the survival rate dropped slowly; while when the dose exceed $1.82 \times 10^{15}$ ions $/ \mathrm{cm}^{2}$, the survival rate increased sharply. But as the dose surpassed $2.34 \times 10^{15}$ ions $/ \mathrm{cm}^{2}$, the survival rate decreased more significantly again. The "down-up-down" pattern curve (also was called "saddle" shape) was consistent with other reports in ion beam implantation ( $\mathrm{Li}$ et al. 2005a), which differed from exponential curves caused by microwave and ultraviolet irradiation ( $\mathrm{Li}$ et al. 2003). Recently, a great number of studies have indicated that the interaction of low energy ions and biological material is more complicated than that of $\gamma$-ray or X-ray radiation with organisms (Yuan 


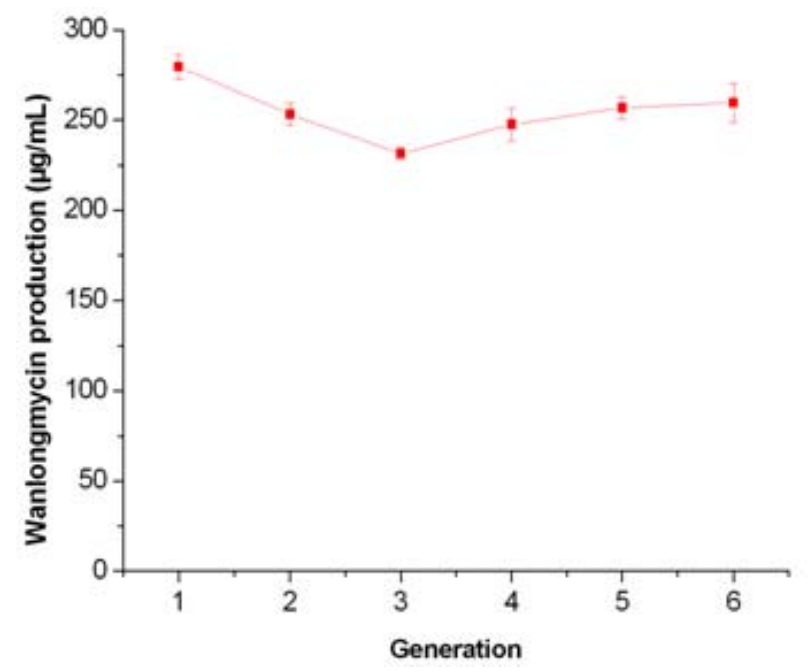

Figure 3. The heredity stability of mutant strain WN939. Data were pooled from three independent experiments. Error bars indicate SEs of mean value.

et al. 2007). Several models have been proposed to explain the "down-up-down" pattern curve, but the mechanisms have not been clarified yet (Song and Yu, 2000).

\section{Mutagenic effects induced by nitrogen ion beam implantation}

The positive mutation rate induced by nitrogen ion beam implantation is shown in Figure 2. The positive mutation rate had close relationship with implantation dose. When the implantation dose was $2.34 \times 10^{15}$ ions $/ \mathrm{cm}^{2}$, the highest positive mutation rate (reached 39.61\%) was achieved, which showed the wide mutation spectrum. The implantation dose of wide mutation spectrum fit the saddle part in the survival curve well.

Usually, the highest mutation rate was obtained when the survival rate of the microorganism was 20 30\% (Su et al. 2006). The results of positive mutation rate by nitrogen ion beam implantation in our experiments validate this viewpoint. Under the conditions of $2.34 \times 10^{15}$ ions $/ \mathrm{cm}^{2}$ implantation dose, one mutant strain WN939 was obtained with a wanlongmycin yield of $271.24 \mu \mathrm{g} / \mathrm{mL}$, which was $82.10 \%$ higher than that of the strain GAAS2507.

In order to ascertain whether it could produce wanlongmycin stably, the mutant strain WN939 was cultivated by flask fermentation for successive generations. Figure 3 showed the wanlongmycin concentration of the second and third generation by flask fermentation decreased slightly; however, the production of the fourth, fifth and sixth generation tended to be stable. Besides, a repeat fermentation was done in a $30 \mathrm{~L}$ fermentor for verification of the stability of the mutant strain WN939. The results (data not shown) indicated the maximum concentration of wanlongmycin in the fermentation processes reached $274.24 \mu \mathrm{g} / \mathrm{mL}$. Both the results showed that the mutant strain WN939 was able to maintain a high yield of wanlongmycin over successive generations.

It had been reported that ionizing radiation could cause a large variety of changes in essential components of cell (Kiefer et al. 1996). In this study, five types of mutant strains (defined as mutant type A, B, C, D, and E strains) were obtained at an energy level of $20 \mathrm{KeV}$ and dose of $2.34 \times 10^{15}$ ions $/ \mathrm{cm}^{2}$, which showed a lot of differences from the wild type strain in colony morphology (Figure 4). Lots of studies suggested that nitrogen ion beam implantation was as different from traditional mutant method, such as X-ray and reagent of chemical mutation, because it has effects of mass deposition and charged ion exchange in addition to energy absorption ( $\mathrm{Li}$ et al. 2005b). Accumulating evidence has shown nitrogen ion implantation technology is a synthesis mutant method including some characteristic of physical and chemical mutation (Yu, 2000).

Table 1 showed the correlation between the changes of colony morphology and production of wanlongmycin, which suggested most mutant type A, B and C strains exhibited positive mutation, while most mutant type $\mathrm{D}$ and E strains exhibited negative mutation. The mutant strain WN939, with the highest yield of wanlongmycin, belonged to mutant type A strain.

\section{CONCLUDING REMARKS}

Nitrogenion beam implantation, characterized by efficiency, safety and non-pollution, seems to be a good way to stimulate the wanlongycin production. The mutant strain WN939 obtained by nitrogenion beam implantation,

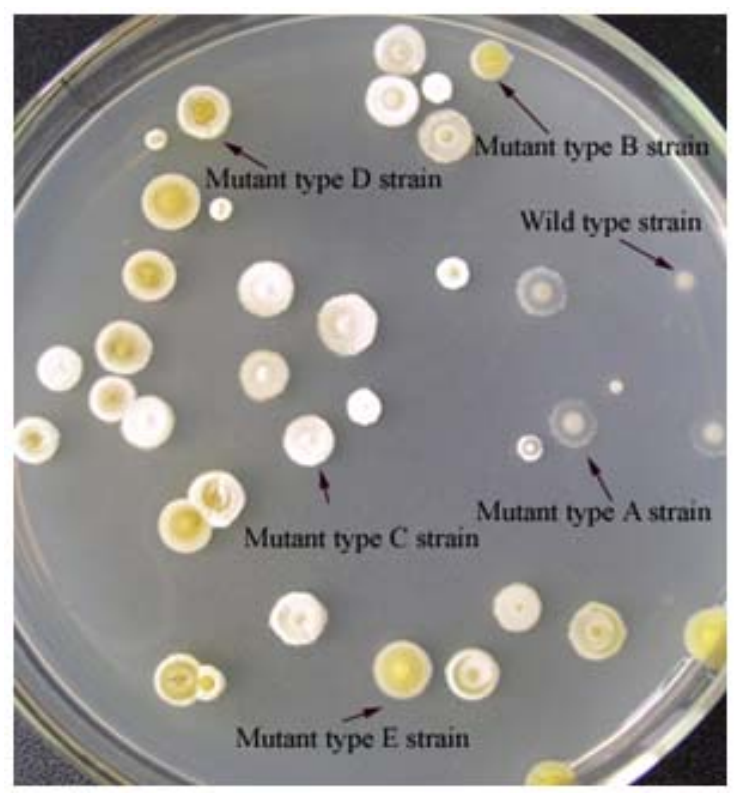

Figure 4. Different colony morphology of strain after GAAS2507 was exposed to nitrogen ions with the energy of $20 \mathrm{KeV}$ and dose of $2.34 \times 10^{15}$ ions $/ \mathrm{cm}^{2}$. 
is $82.10 \%$ higher yield than that of the wild strain GAAS2507 and is able to perform stably in batch fermentation. Five types of mutant strains, isolated under the condition of the energy level of $20 \mathrm{KeV}$ and dose of $2.34 \times 10^{15}$ ions $/ \mathrm{cm}^{2}$, show a lot of differences from the wild type strain including the production of wanlongmycin and colony morphology. On the basis of the correlation between the colony morphology and the level of wanlongmycin production of mutant strains, a fast and effective method of screening wanlongmycin high-yield strains has been developed in here, which provides a good foundation for further investigation and research on screening industrial strains with higher yield of wanlongmycin.

\section{ACKNOWLEDGMENTS}

The authors are grateful to Pref. Wu Yuejin of Key Laboratory of Ion Beam Bioengineering, Chinese Academy of Sciences, China, for his kindness in facilitating the ion beam bioengineering instrument.

\section{REFERENCES}

DU, Yanhua; HUANG, Shenghai; TAN, Zheng; LIN, Yingzhi and QIU, Guanying. Dertermination of DNA single-strand breaks by low-energy heavy ion and analysis of dose effect curves. Chinese Science Bulletin, April 1999, vol. 44, no. 8, p. 711-715.

GU, Shao-bin; LI, Shi-chang; FENG, Hui-yun; WU, Ying and YU, Zeng-liang. A novel approach to microbial breeding-low energy ion implantation. Applied Microbiology and Biotechnology, January 2008, vol. 78, no. 2, p. 201-209.

KIEFER, Jürgen; BREND’AMOUR, Mathias and STOLL, Uwe. Heavy ion action on biological systems. Nuclear Instruments and Methods in Physics Research Section B: Beam Interactions with Materials and Atoms, February 1996, vol. 107, no. 1-4, p. 292-298.

LI, Yongquan; CHEN, Shifei and CEN, Peilin. Mutagenesis and screening of high yield xylanase production strain of aspergillus usami by microwave irradiation. Chinese Journal of Chemical Engineering, October 2003, vol. 11, no. 5, p. 594-597.

LI, Shichang; WU, Min; YAO, Jianming; PAN, Renrui and YU, Zengliang. Mutation-screening in xylanase-producing strains by ion implantation. Plasma Science and Technology, February 2005a, vol. 7, no. 1, p. 2697-2700.

LI, Guoping; HUANG, Qunce; QIN, Guangyong and HUO, Yuping. The effects of low-energy nitrogen ion implantation on pollen exine substructure and pollen germination of cedrus deodara. Plasma Science and Technology, December 2005b, vol. 7, no. 6, p. 3176-3180.
LIU, Jing and YAO, Jianming. Study on mutagenic breeding of bacillus subtilis and properties of its antifungal substances. Plasma Science and Technology, August 2004, vol. 6, no. 4, p. 2433-2436.

LIU, Qingmei; YUAN, Hang; WANG, Jun; GONG, Guohong; ZHOU, Wei; FAN, Yonghong; WANG, Li; YAO, Jianming and YU, Zengliang. A mutant of bacillus subtilis with high-producing surfactin by ion beam implantation. Plasma Science and Technology, July 2006, vol. 8, no. 4, p. 491-496.

MULLER, Hermann Joseph. Artificial transmutation of the gene. Science, July 1927, vol. 66, no. 1699, p. 84-87.

SHI, Huai-bin; SHAO, Chun-lin and YU, Zeng-liang. Dose effect of keV ions irradiation on adenine and cytosine. Acta Biophysica Sinica, October 2001, vol. 17, no. 4, p. 731-735.

SONG, Dao-jun and YU, Zeng-liang. Etching and damage action on microbes' cells by low energy $\mathrm{N}^{+}$beam. Plasma Science and Technology, August 2000, vol. 2, no. 4, p. 415421.

SU, Caixin; ZHOU, Wei; FAN, Yonghong; WANG, Li; ZHAO, Shiguang and YU, Zengliang. Mutation breeding of chitosanase-producing strain Bacillus sp. S65 by lowenergy ion implantation. Journal of Industrial Microbiology and Biotechnology, August 2006, vol. 33, no. 12, p. 1037-1042.

WU, L. and YU, Z. Radiobiological effects of low-energy ion beam on wheat. Radiation and Environmental Biophysics. March 2001, vol. 40, no. 1, p. 53-57.

XU, An; YAO, Jingmin; YU, Lixiang; WANG, Jun and YU, Zengliang. Mutation of Gluconobacter oxydans and Bacillus megaterium in a two-step process of L-ascorbic acid manufacture by ion beam. Journal of Applied Microbiology, February 2004, vol. 96, no. 6, p. 1317-1323.

YANG, Ying-ge; FAN, Yong-hong; LI, Wen; WANG, Dong-mei; WU, Yue-jin; ZHENG, Zhi-ming and YU, Zeng-liang. Optimization of $\mathrm{L}(+)$-lactic acid production from xylose with rhizopus oryzae mutant RLC41-6 breeding by low-energy ion implantation. Plasma Science and Technology, October 2007, vol. 9, no. 5, p. 638-642.

YU, Zeng-liang, WANG, Xuedong and DENG, Jianguo. Primary studies of mutational mechanism for rice induced by ion implantation. AnHui Agriculture Science, 1989, vol. 28, no. 1, p. 12-16.

YU, Zeng-liang. Interaction between low energy ions and the complicated organism. Plasma Science and Technology, December 1999, vol. 1, no. 1, p. 79-85.

YU, Zeng-liang. Ion beam application in genetic modification. IEEE Transaction on Plasma Science, February 2000, vol. 28, no. 1, p. 128-132. 
YUAN, Hang; ZHOU, Wei; WANG, Jun; ZHANG, Shuqing and YAO, Jianming. Enhancement of gongronella sp. JG chitosanase production by ion beam implantation. Plasma Science and Technology, February 2007, vol. 9, no. 1, p. 115-118.

ZHAO, Yong; TAN, Zheng; DU, Yan-hua and QIU, Guanying. Electrophoresis examination of strand breaks in plasmid DNA induced by low-energy nitrogen ion irradiation. Nuclear Instruments and Methods in Physics Research Section B: Beam Interactions with Materials and Atoms, October 2003, vol. 211, no. 2, p. 211-218.

ZHANG, Xiaoyong; LIN, Birun; GAO, Xiangyang and SHEN, Huifang. Determination of wanlongmycin by high performance liquid chromatography. Chinese Journal of Biological Control, October 2006, vol. 22, no. s1, p. 78-81.

ZHOU, Xiuhong; PAN, Renrui; GE, Chunmei; YAO, Jianming and YU, Zengliang. Optimization of L-lactic acid production of Rhizopus oryzae mutant RLC41-6 by ion beam implantation at low-energy. Plasma Science and Technology, October 2005, vol. 7, no. 5, p. 3078-3080. 